\title{
Síntese de Compostos Orgânicos por via Electroquímica
}

\author{
A NA PAULA PAIVA *
}

\begin{abstract}
A sintese electroquímica de compostos orgânicos é um ramo da ciência já com cerca de 150 anos, ao longo dos quais extensa actividade científico-laboratorial tem sido empreendida. Pretende-se, com este trabalho, divulgar as particularidades e vantagens de aplicação desta metodologia. Após um breve relato da respectiva evolução histórica, apresentam-se genericamente as características essenciais das reacções electroorgânicas em solução, ilustradas com exemplos significativos. A projecção actual e perspectivas futuras de aplicação industrial desta alternativa de síntese são também analisadas e discutidas.
\end{abstract}

\section{INTRODUÇÃO}

A primeira reacção de síntese orgânica envolvendo o uso de electricidade levou à formação de ligações carbono-carbono, as quais se podem considerar quase como que a "essência" da química orgânica. Com efeito, as observações preliminares de Faraday, em 1834, de que havia evolução de etano e dióxido de carbono durante a electrólise de soluções aquosas de sais de ácido acético [1], levaram Kolbe a estudar as potencialidades de aplicação de tal descarboxilação electrolítica [2], o que conduziu ao estabelecimento dos patamares da síntese electroorgânica que ainda actualmente são bastante utilizados e cujo procedimento ainda guarda o nome do seu descobridor reacção de Kolbe.

Kolbe electrolisou soluções aquosas de semi-ésteres de ácidos gordos e verificou a formação de hidrocarbonetos de cadeia longa, resultante da dimerização dos respectivos esqueletos hidrocarbonados, acompanhada de descarboxilação [2]. O mecanismo desta reacção tem sido alvo de discussão em anos recentes, devido essencialmente aos avanços de conhecimento acerca dos fenómenos de electroadsorção frequentemente associados a estes processos $[3,4]$.

A reacção de Kolbe é apenas um exemplo bem conseguido de uma síntese electroorgânica que foi possível levar a cabo com os conhecimentos científicos do século passado. Promover a passagem de uma corrente eléctrica, mesmo constante, através de soluções contendo compostos orgânicos, dificilmente conduz à formação selectiva dos produtos esperados. Assim, os esforços de investigação empreendidos no estudo de electrólises de soluções de compostos orgânicos, envolvendo a aplicação de corrente eléctrica constante e reactores muito simples, encontram-se compilados num livro da autoria de F. Fichter [5], da década de 40 , donde sobressai, na maior parte dos casos, a grande complexidade das misturas reaccionais resultantes de tais tratamentos.

A década de 60 assistiu a um grande avanço na implementação do método, como consequência directa do desenvolvimento das técnicas electroanalíticas. Com efeito, data desta época uma intensificação dos estudos de determinação de potenciais de oxidação e redução de variadas moléculas orgânicas [6]. Houve também um interesse crescente na investigação das características de radicais iónicos, espécies intermediárias importantes em oxidações e reduções, nomeadamente após a descoberta de que a redução de Birch envolvia espécies radicalares do tipo aniónico. No entanto, o grande impulso de divulgação que o método teve na época ficou a dever-se ao início da produção industrial, em larga escala, de adiponitrilo a partir da electrohidrodimerização de acrilonitrilo, emprendida pela firma Monsanto em 1964 - 1965 [6,7]. O processo ainda se mantém em laboração actualmente, apesar de ter vindo a sofrer um número considerável de ajustes ao longo do tempo, tendentes a rentabilizá-lo o mais possível [7].

O sucesso comercial da referida electrossíntese suscitou o interesse quer de químicos orgânicos de síntese quer de electroquímicos, e o progresso desses esforços, inicialmente bastante lento devido ao baixo nível de conhecimento sobre os mecanis- mos das reacções electroorgânicas, tem vindo mais recentemente a ser significativo, particularmente nos últimos dez anos [6]. Todavia, sendo necessária uma colaboração estreita entre estas duas áreas da química para um avanço efectivo deste ramo científico, têm sido essencialmente os estudos de carácter electroquímico que mais abundam, pelo que os objectivos electrossintéticos propriamente ditos são frequentemente ultrapassados pela investigação de aspectos exclusivamente mecanísticos.

Não pretendendo ignorar a importância da via electroquímica em fase sólida-gasosa na obtenção dos denominados fulerenos e derivados de inclusão com eles relacionados [8], é objectivo deste trabalho a apresentação dos principais tipos de reacções electroorgânicas em solução e divulgar os aspectos mais recentes da sua implementação.

\section{REACÇÕES \\ ELECTROORGÂNICAS DIRECTAS}

Este tipo de reacção inicia-se por uma transferência electrónica directa entre espécies em solução e um eléctrodo. Tal transferência electrónica provoca a oxidação ou redução do substracto orgânico, originando intermediários reactivos que, consoante o processo envolvido, são de diferente natureza - Figura 1.

São estes intermediários que, no seio da mistura reaccional, reagem entre si para originarem os produtos de síntese - há casos, no entanto, em que os intermediários reagem enquanto adsorvidos à superfície do eléctrodo [9].

A escolha do potencial eléctrico a aplicar no eléctrodo de trabalho é extremamente importante, uma vez que é este parâmetro electroquímico que determina a extensão e a velocidade da transferência electrónica substracto-eléctrodo; todavia, no planeamento de uma electrossíntese orgânica, para haver selectividade, é também necessário um controlo criterioso e imaginativo da química dos 


\section{Substrato orgânico R}

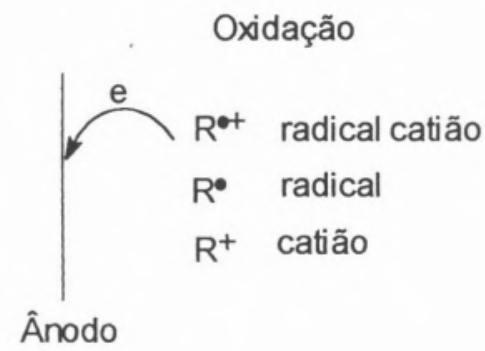

Oxidação

$\mathrm{R}^{\bullet}$ radical
$\mathrm{R}^{+}$catião

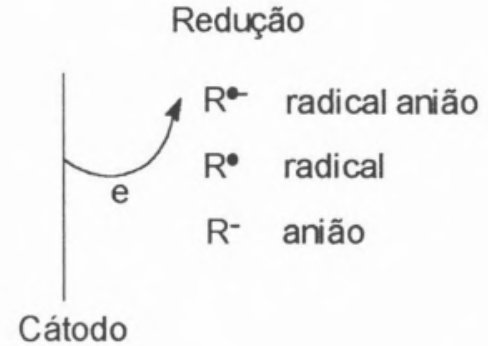

Fig. 1. - Tipos fundamentais de intermediários reactivos durante os processos de oxidação e redução electródica.

intermediários reactivos que se formam [9], pelo que o estudo dos processos electrossintéticos deste tipo é, sem dúvida, um desafio fascinante.

\section{Exemplos de reduções catódicas directas}

Incluído no vasto grupo das electroreduções de alcenos que originam hidrodímeros, o processo Monsanto de transformação do acrilonitrilo em adiponitrilo merece especial referência. Assim, a respectiva reacção catódica pode ser representada por (1),

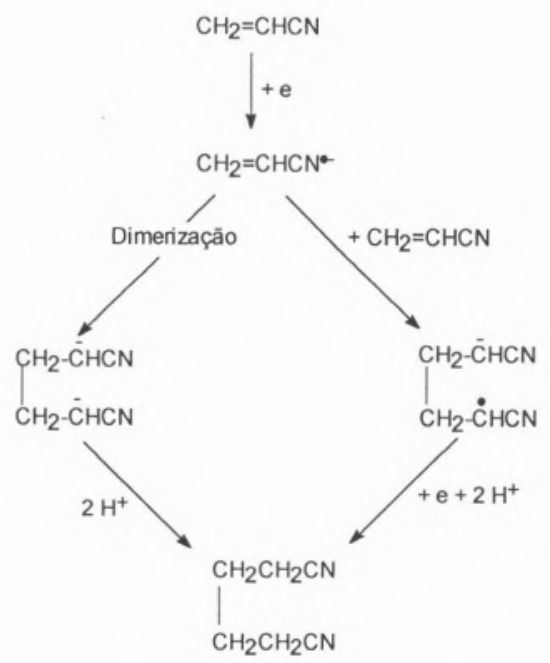

Fig. 2. - Dois dos mecanismos possíveis para a electrohidrodimerização do acrilonitrilo a adiponitrilo. sendo dois dos possíveis mecanismos de electroredução do substracto orgânico apresentados na Figura 2.

$$
\begin{gathered}
2 \mathrm{H}_{2} \mathrm{C}=\mathrm{CH}-\mathrm{CN}+2 \mathrm{e}+2 \mathrm{H}^{+} \rightarrow \\
\mathrm{NC}-\left(\mathrm{CH}_{2}\right)_{4}-\mathrm{CN}
\end{gathered}
$$<smiles>COc1ccc2cc(C(C)C(=O)O)ccc2c1</smiles>

Fig. 3. - Exemplos de carboxilações catódicas.
Para além dos referidos, há ainda possibilidade de ocorrência de outros patamares mecanísticos; por exemplo, tem sido também considerada a formação do dianião de acrilonitrilo (ACN) por transferência de
2 electrões, prosseguindo a sequência reaccional com a dimerização por acoplamento com uma molécula neutra de ACN e subsequente protonação do dianião resultante [10]. No entanto, há ainda diversos outros mecanismos possíveis que podem eventualmente coexistir [7], dependendo provavelmente das condições experimentais específicas adoptadas. Pormenores recentes do processo industrial em si - em termos do tipo de célula e eléctrodos, electrólito suporte, eficiência e selectividade de produção de adiponitrilo, eficiência de corrente -, estão disponíveis na literatura especializada $[7,10]$.

A redução catódica de moléculas orgânicas origina usualmente carbaniões que podem reagir selectivamente com um agente electrófilo, por exemplo, dióxido de carbono, provocando-se deste modo uma “carboxilação catódica". É esta reacção que é seguida para a síntese de

Naproxen<smiles>COc1ccc2cc(C(C)C(=O)OCc3ccc4cc(C(C)Cl)ccc4c3)ccc2c1</smiles><smiles>COc1ccc2cc(C(C)=O)ccc2c1</smiles>

drogas anti-inflamatórias, por exemplo, do denominado "naproxen", a partir de um derivado cloretado [11] e de uma cetona [12], Figura 3. Estas reacções já foram testadas à escala piloto [9]. 
Outro tipo importante de redução catódica em moléculas orgânicas são as chamadas "reaç̧ões de clivagem", muito utilizadas na eliminação de grupos protectores e que levam à formação de substractos de modo selectivo. Esta tem sido uma importante área de investigação na Universidade do Minho [13,14]. Por outro lado, a redução catódica da L-cistina produz a cisão da sua ligação enxofre-enxofre e a formação da L-cisteína, processo que também já foi testado à escala piloto, com resultados prometedores $[9,15]$.

\section{Exemplos de oxidações anódicas directas}

Um exemplo imprescindível de uma oxidação anódica directa é, como se referiu na Introdução, a síntese de Kolbe. Por estranho que pareça, esta reacção continua a ser a única síntese electroorgânica a merecer referência em alguns livros de texto de química orgânica geral mais usuais, tais como o de Graham Solomons [16], autor mundialmente consagrado, mas não é mesmo sequer mencionada em muitos outros.

A tecnologia necessária para a oxidação anódica do monoadipato de metilo (na forma de sal alcalino) a sebacato de dimetilo - intermediário importante na síntese do nylon 6,10 e para a indústria da borracha -, está perfeitamente dominada por algumas empresas industriais $[6,10]$, mas o processo ainda não é explorado comercialmente [10]. A reacção geral que sumaria a transformação é a (2), apresentando-se de seguida - Figura 4 - o mecanismo geral associado.

$$
\begin{gathered}
2 \mathrm{H}_{3} \mathrm{COOC}-\left(\mathrm{CH}_{2}\right)_{4}-\mathrm{COO}^{-}-2 \mathrm{e} \rightarrow \\
\mathrm{H}_{3} \mathrm{COOC}-\left(\mathrm{CH}_{2}\right)_{8}-\mathrm{COOCH}_{3}+2 \mathrm{CO}_{2}
\end{gathered}
$$

Em termos de síntese de novos produtos, é a síntese de Kolbe mista que presentemente tem sido mais investigada $[1,4,9]$. Com efeito, usando dois ácidos carboxílicos parcialmente neutralizados, a sua oxidação anódica directa resultará na mistura

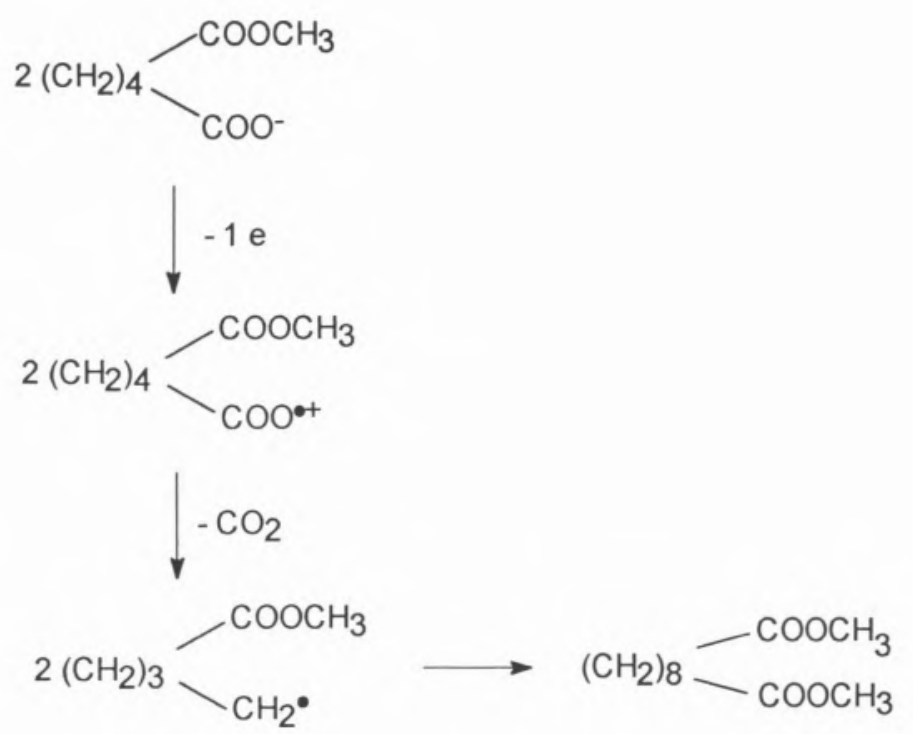

Fig. 4. - Mecanismo geral associado à síntese de Kolbe do sebacato de dimetilo.

estatística de três dímeros, (3), cujas concentrações se podem controlar de modo a favorecer a síntese do dímero misto.

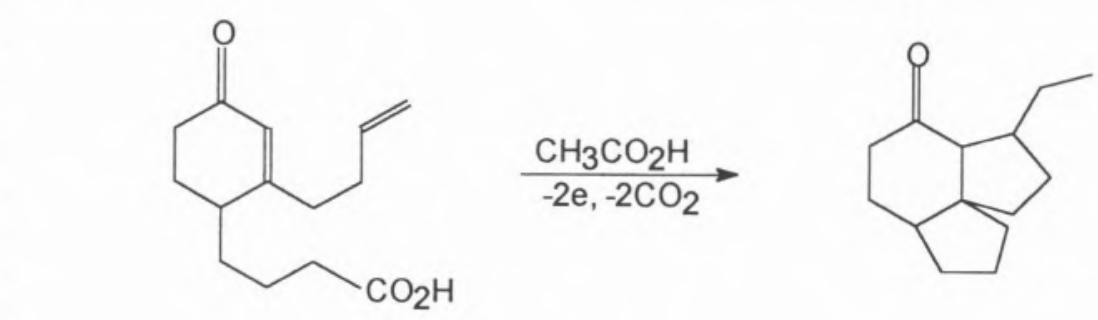

síntese são as metoxilações. Para isso, promove-se a oxidação anódica directa de um substrato orgânico a um radical catião que, dissolvido em

Fig. 5. - Um exemplo de uma reacção de Kolbe precedida por um rearranjo intramolecular.

$$
\begin{array}{r}
\mathrm{RCO}_{2}^{-}+\mathrm{R}^{\prime} \mathrm{CO}_{2}^{-}-2 \mathrm{e} \rightarrow \mathrm{R}-\mathrm{R}+\mathrm{R}- \\
\mathrm{R}^{\prime}+\mathrm{R}^{\prime}-\mathrm{R}^{\prime}
\end{array}
$$

$\mathrm{R}, \mathrm{R}^{\prime}$ - grupos substituintes

Outra perspectiva útil neste tipo de síntese é a que resulta do rearranjo intramolecular rápido que pode ocorrer antes do acoplamento, apresentando-se na Figura 5 um exemplo relativamente recente em que esta potencialidade é explorada [9].

Um outro tipo de oxidação com grandes potencialidades em electros- metanol, vai imediatamente reagir com o solvente, ao que se seguem a perda de outro electrão e protão para originar o produto final $[4,9]$. Na Figura 6 são apresentados dois exemplos deste tipo de reacção $[6,9,17,18]$. A transformação do p-cresol a 4-hidroxi-benzaldeído é um processo industrial em curso [6,9], que ilustra a necessidade de utilização de grupos protectores para evitar reacções indesejáveis, procedimento muito à semelhança do que se utiliza em química orgânica convencional. 
Pelo interesse que despertou industrialmente, tendo já sido uma via processual várias vezes testada à escala piloto [10], é pertinente referir a oxidação anódica do benzeno a benzoquinona e posterior redução catódica deste intermediário a hidroqui-<smiles>c1ccccc1</smiles>

adsorção indesejada de espécies à superfície dos mesmos, pelo que estes inconvenientes também podem ser evitados por utilização do método indirecto. Adicionalmente, quando a adopção do uso do sistema redox é possível, as reacções acopladas ocor-

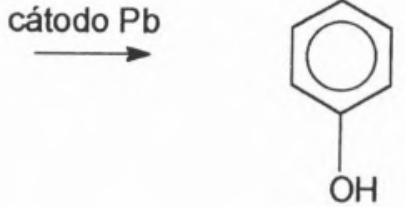

nona, de acordo com o esquema apresentado em (4).

Para esta transformação electroquímica é proposto o uso de uma célula de electrólise equipada com uma membrana de troca catiónica e eléctrodos de óxido de chumbo e de chumbo para ânodo e cátodo, respectivamente [10].

\section{REACÇÕES \\ ELECTROORGÂNICAS INDIRECTAS}

As reacções electroorgânicas indirectas ocupam um lugar de destaque cada vez maior no panorama da electrossíntese de moléculas orgânicas. O método baseia-se na possibilidade de utilização de um sistema redox que, por transferência electrónica com um eléctrodo, adquire a capacidade de oxidar ou reduzir o substrato orgânico, sendo depois novamente regenerado por novo contacto electródico. Assim sendo, o sistema redox vai sendo sucessivamente reciclado o que faz com que, na essência, funcione como um catalisador [4,17]. Esta é, aliás, uma das grandes vantagens das electrossínteses indirectas: não é necessário o uso estequiométrico de reagentes tóxicos e/ou excessivamente caros os quais, mesmo se usados no sistema redox, não produzem efluentes. Por outro lado, há reacções directas que se inibem por corrosão dos eléctrodos ou rem, em geral, a um potencial eléctrico menor do que o necessário para as correspondentes electrossínteses directas [6]. do p-cresol a 4-hidróxi-benzaldeído $[6,8]$.

\section{Exemplos de reacções electroorgânicas indirectas}

Também aqui há já alguns processos usados industrialmente e outros com grande probabilidade de o virem a ser.

Um dos tipos de electrossintese indirecta que se tem vindo a utilizar com bastante sucesso é o que se baseia no sistema redox $\mathrm{Ce}$ (III) / Ce(IV) em solução aquosa de ácido metano-sulfónico, que permite quer a oxidação selectiva de toluenos a benzaldeídos, quer de naftalenos e antracenos às correspondentes quinonas $[19,20]$. Está previsto para 1998 o início da laboração de um complexo industrial no Canadá que, baseado neste processo, promoverá a oxidação do naftaleno a naftaquinona, intermediário em seguida sub-
1)<smiles>Cc1ccc(O)cc1</smiles><smiles>COCc1ccc(C)cc1</smiles><smiles>COCc1ccc(C)cc1</smiles><smiles>Cc1ccc(C=O)cc1</smiles>

2)<smiles>Cc1ccc(O[C@@H](C)[PH2+][C@H](C)O)cc1</smiles><smiles>CC(C)c1ccc(OC(C)(C)C)cc1</smiles>

Fig. 6. - Dois exemplos de metoxilações: 1) conversão do p-xileno a p-metil-benzaldeído [16,17]; 2) conversão 
metido a uma reaç̧ão de Diels-Alder com butadieno para originar a antraquinona - Figura 7 - [9], composto muito utilizado como catalisador na indústria do papel [6].

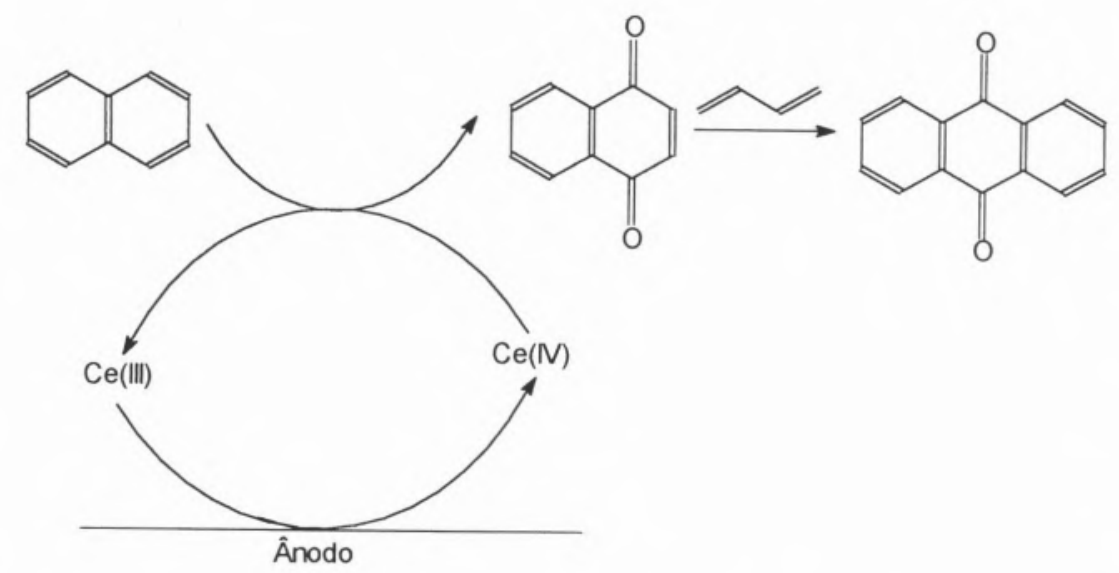

Fig. 7. - Conversão do naftaleno a antraquinona envolvendo uma electrossíntese indirecta.
Outro exemplo relevante e de interesse para a indústria farmacêutica é o da oxidação de alcenos a dióis opticamente activos, em que a alternativa electroquímica $[21,22]$ também usa as condições experimentais propostas por Sharpless [23] para esta conversão. O sistema redox é, para este caso, um complexo de ósmio com um ligando quiral emulsão aquosa-orgânica. Assim, o complexo é que vai oxidar o substracto orgânico, sendo depois continuamente regenerado, na interface aquosa-orgânica, pelo ião ferricianeto que, por sua vez, é regenerado no ânodo, Figura 8.

Vantagem nítida conseguida pela adopção da electrossíntese indirecta nos dois exemplos acima referidos É a possibilidade de uso de pequenas quantidades de sistema redox (contrariamente à via convencional, que requereria quantidades estequiométricas): o catalisador $\mathrm{Ce}$ (III) / Ce(IV) é muito caro [6] e o de ósmio é extremamente tóxico [9]. Por outro lado, sendo os sistemas redox continuamente regenerados no processo, pode considerarse que as electrossínteses indirectas são vias processuais "limpas".

\section{CONCLUSÕES}

Tendo sido dada uma perspecticomo o 4-clorobenzoato de hidroquinidina [21]. Esta electrossíntese indirecta é ainda mais elaborada do que as usuais, na medida em que utiliza dois sistemas redox e uma

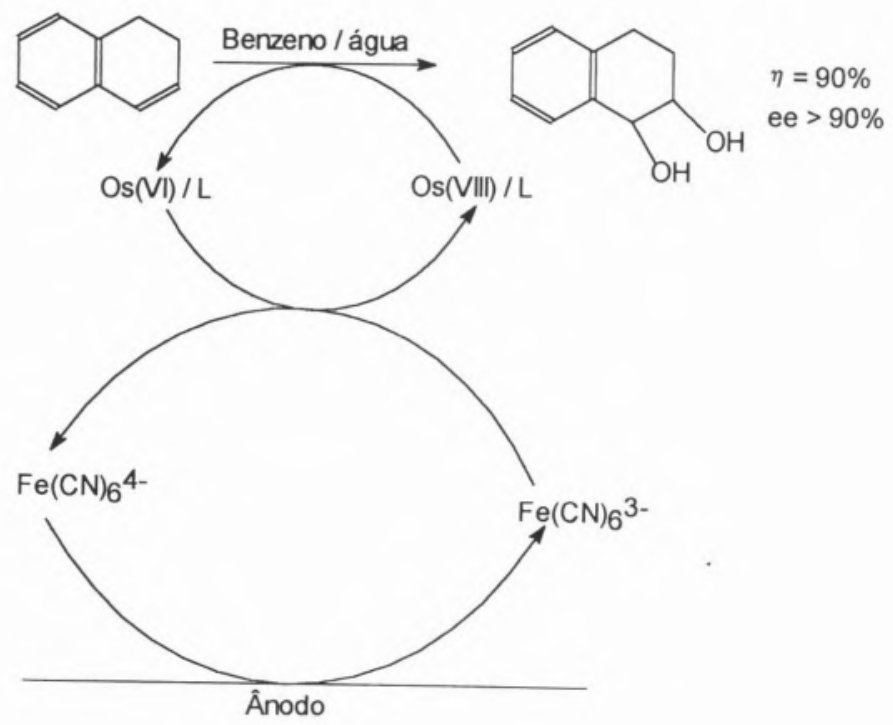

Fig. 8. - Oxidação anódica indirecta de alceno a diol por utilização de dois sistemas redox. 
iii) Relativamente à implantação industrial, apesar de já haver diversas indústrias a incluir reacções electrorgânicas, há ainda um número considerável de processos, já comprovados à escala piloto como economicamente viáveis, mas que não foram ainda adoptados à escala comercial. Tal situação fica essencialmente a dever-se a factores económicos associados às dificuldades em modificar, de raíz, sectores de complexos industriais que não usam a via electroquímica em nenhuma fase processual. No entanto, uma perspectiva a médio prazo para a implementação deste ramo científico à escala industrial é, sem dúvida, optimista.

* CECUL, Departamento de Química e Bioquímica. Faculdade de Ciências da Universidade de Lisboa, Rua Ernesto de Vasconcelos, C1, $5^{\circ}$ Piso, 1700 Lisboa
REFERÊNCIAS BIBLIOGRÁFICAS

1. D. Kyriacou, Modern Electroorganic Chemistry, Springer-Verlag Lab. Manual (1994).

2. H. Kolbe, Leibigs Ann. 69 (1849) 257.

3. J. A. Bockris, S. Khan, Surface Electrochemistry, Plenum Press, Nova lorque (1993)

4. A. I. Fry, Synthetic Organic Electrochemistry, John Wiley and Sons, $2^{\mathrm{a}}$ ediçào, Nova lorque (1989).

5. Fr. Fichter, Organische Elektrochemie, Theodor Steinkopff, Dresden (1942).

6. I. Utley, Chemistry and Industry, 6 (1994) 215.

7. D. Pletcher, F. C. Walsh, Industrial Electrochemistry, Capítulo 6, Chapman and Hall, Londres, (1990).

8. M. N. Berberan e Santos, Revista Portuguesa de Química 3 (1996) 3, e referências aí citadas.

9. J. D. Genders, D. Pletcher, Chemistry and Industry, 18 (1996) 682

10. K. Weissermel, H.-J. Arpe, Industrial Organic Chemistry, VCH, $2^{3}$ edição, Weinheim, (1993).

11. I. Chaussard, I. -C. Folest, I. Y. Nédélec, I. Perichon, S. Sibelle, M. Troupel, Synthesis, (1990) 369.

12. D. Pletcher, L. Slevin, I. Chem. Soc. Perkin Trans. 2 2 (1996) 217.
13. M. I. Montenegro, Electrochim. Acta, 31 (1986)

14. H. L. S. Maia, L. S. Monteiro, F. Degerbeck, L. Grehn, V. Ragnarsson, 1. Chem. Soc. Perkin Trans. 2 3 (1993) 495.

G. Sancho-Cano, V. Montrel, A. Aldaz, Tetrahedron, 47 (1991) 1067.

16. G. Solomons, Organic Chemistry, 6a Edição, John Wiley and Sons, Nova lorque, (1996).

17. T. Shono, Electroorganic Synthesis, Academic Press Ltd, Londres, (1991).

18. L. M. Abrantes, A. P. Paiva, "Estudo da oxidação anódica directa do p-xileno", comunicação apresentada no $15^{\circ}$ Encontro da Sociedade Portuguesa de Química, Porto, Maio de 1996

19. R. P. Kreh, R. M. Spotnitz, J. T. Lundqvist, Tetrahedron Lett. 28 (1987) 1067

20. R. P. Kreh, R. M. Spotnitz, J. T. Lundqvist, I. Org. Chem., 54 (1989) 1526.

21. A. R. Amundsen, E. N. Balko, I. Appl. Electrochem. 22(9) (1992) 810.

22. S. Torii, P. Lui, H. Tanaka, Chem. Lett., (1995) 319.

23. E. N. Jacobsen, I. E. Marko, W. S. Mungall, G Schroder, K. B. Sharpless, I. Am. Chem. Soc. 110 (1988) 1968.

\section{Espectrofotómetro de Absorção Atómica Modelo AAS5 EA \\ da Nossa Representada "ANALYTIK JENA"}
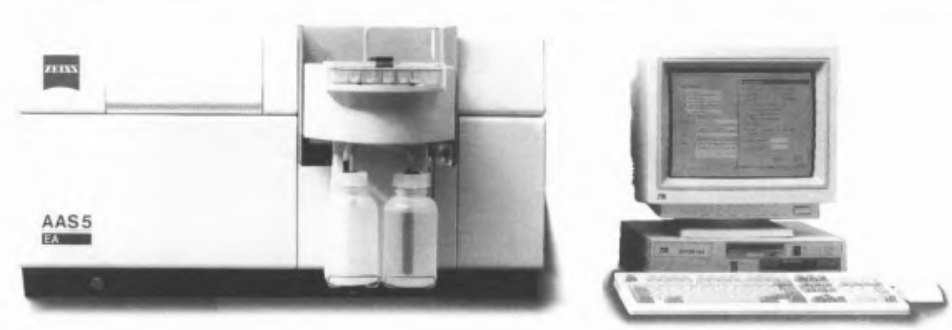

Espectrofotómetro de Absorção Atómica, completamente automático, com Câmara de Grafite para análise de amostras reais em matrizes complexas. A combinação do tubo de grafite aquecido transversalmente, com a poderosa óptica Carl Zeiss e com um software eficiente permite um rendimento analítico excelente com níveis de detecção, precisão e reprodutibilidade de primeira classe.

- Rápida correcção do ruído de fundo $D_{2}$.

- Análise multi-elemento automática.

- Vários tubos e plataformas para garantir todas as técnicas analíticas.

- Aquecimento super rápido para assegurar um tempo de análise mínimo.

Sociedade de Representações e Química, Lda.

Rua Coronel Santos Pedroso, 15 • 1500 LISBOA • Tel.: 7165160 • Fax: 7165169

Sede Social: Av. da Liberdade, 220-2 $2^{\circ} 129$ LISBOA CODEX

Rua 5 de Outubro, $269 \bullet 4100$ PORTO • Tels.: $6093069 \bullet$ Fax: 6000834

Email: soquimica@mail.telepac.pt;Internet:www.soquimica.pt 